\title{
Colossal magnetoresistance and anomalous Hall effect in nonmagnetic semiconductors
}

\author{
Sergei Obukhov ${ }^{1}$, and Tatiana Panysheva ${ }^{2}$ \\ ${ }^{1}$ Ioffe PTI of the RAS, 194021, St. Petersburg, Russian Federation \\ ${ }^{2}$ Peter the Great St. Petersburg Polytechnic University, 194064, St. Petersburg, Russian Federation
}

\begin{abstract}
Colossal Magnetoresistance (CMR) in nonmagnetic semiconductors and magnetic materials has been investigated as a function of magnetic field, charge carriers concentration and temperature. Both types of materials demonstrated qualitative and quantitative coincidence of CMR dependence on magnetic field, charge carriers concentration and temperature. The findings support the CMR interpretation in the framework of the Excitonic Insulator (EI) model and transition of an insulating El phase to conducting state under magnetic field for all types of materials under study. It is suggested that Jahn-Teller distortion caused by magnetic ions and external uniaxial stress could initiate El phase formation
\end{abstract}

\section{Introduction}

The effect of specific resistance decrease in magnetic field, known as Negative Magnetoresistance (NM), was first observed by Lord Kelvin in ferromagnetic metals in 1856 [1]. Still the numerous attempts to reveal the nature of the NM effect in most types of solid state materials, like semiconductors, superconductors, perovskites, graphite remain debatable. Nowadays NM phenomenon exploration in the above materials has been boosted by the practical interest as their characteristics meet the demands of hard disk driver technology, spintronics and magnetoelectronics [2].

Special attention was attracted to NM effect in Magnetic Semiconductors (MS) like $n-G_{3-x} \mathrm{v}_{x} \mathrm{~S}_{4}$ [3], Diluted Magnetic Semiconductor (DMS) $\mathrm{Hg}_{1-\mathrm{x}} \mathrm{Mn}_{\mathrm{x}} \mathrm{Te}$ [4] and Manganite Perovskite like $\operatorname{Pr}_{0,7} \mathrm{Ca}_{0,3} \mathrm{MnO}_{3}$ [5], all of which demonstrated colossal resistivity decrease in external magnetic field, the effect known as Colossal Magnetoresistance (CMR).

The physical models describing CMR, i.e. Magnetic Polaron and Phase Separation models [6,7] are based on the suggestion that MS and Manganite Perovskite are magnetic materials, where charge carriers transport is dependent on the interaction with the magnetic moments of the host magnetic ions.

However, this approach absolutely ignores the fact that CMR has been also experimentally observed in nonmagnetic semiconductors (NMS), i.e. p-Ge $[8,9]$ and p-InSb $[8,10]$, where resistivity decrease of $10^{2} \div 10^{5}$ times was demonstrated in magnetic field $\mathrm{B} \sim 3 \mathrm{~T}$ and temperature $\mathrm{T} \sim 1,5 \mathrm{~K}$, thus at the experimental conditions similar to the conditions applied to MS, DMS and Manganite Perovskite in CMR studies.
In this paper we aim to conduct the comparative study based on our magnetotransport experimental findings revealed for nonmagnetic semiconductors and the results of magnetic CMR materials studies described in scientific literature for the similar experimental conditions. This approach can help to expand CMR understanding and establish links between these two groups of solid state materials.

\section{CMR comparison in nonmagnetic and magnetic materials}

In this section we compare our experimental results of the CMR effect studies in NMS as the function of magnetic field, charge carrier concentration and temperature with the data adopted from literature for DMS, MS and Manganite Perovskite [3-5,12].

\subsection{Experimental}

p-InSb(Ge), p-InSb(Mn) and p-Ge(Ga) crystals were grown by Czochralski method. Magnetoresistance, the Hall effect and dc conductivity were measured at Hall bar samples $7 \times 1.5 \times 1 \mathrm{~mm}^{3}$. The materials were studied under the low and high magnetic field $(B=0$ $15 \mathrm{~T}$ ), temperature $\mathrm{T}=40 \mathrm{mK}-4 \mathrm{~K}$ and charge carrier concentration $\mathrm{n}=10^{16} \div 10^{18} \mathrm{~cm}^{-3}$. Measurements at superlow temperatures were carried out in ${ }^{3} \mathrm{He}-{ }^{4} \mathrm{He}$ dilution refrigerator with $15 \mathrm{~T}$ Oxford Instruments superconductive magnet at NHMFL, Florida. Measurements in the temperature range $\mathrm{T}=300 \div 1,5 \mathrm{~K}$ were carried out on 15T Bitter magnet of HMFL in Technical University Braunschweig, Germany. ${ }^{3} \mathrm{He}$ cryostate at $\mathrm{T}=$ $4 \div 0,3 \mathrm{~K}$ with $7 \mathrm{~T}$ superconductive magnet was used for

* Corresponding author: author@e-mail.org 
magnetotransport measurements at The Institute of Physics, The University of Sao Paulo, Brasil.

\subsection{CMR dependence on magnetic field}

The dependence of CMR value on magnetic field strength in NMS, DMS, MS and Manganite Perovskite is demonstrated in figure 1 . The curves represent data for the following CMR materials:

a. (NMS) uniaxially stressed InSb crystal doped with Ge at $1,84 \mathrm{~K}$; InSb crystal doped with $\mathrm{Mn}$ at $40 \mathrm{mK}$ and $\mathrm{Ge}$ crystal doped with Mercury at $\mathrm{T}=35 \mathrm{~K}$, adopted from [9]

b. (DMS) $\mathrm{p}-\mathrm{Hg}_{1-\mathrm{x}} \mathrm{Mn}_{\mathrm{x}} \mathrm{Te}$ at $1,7 \mathrm{~K}$ [4]

c. (MS) $n-\mathrm{Gd}_{3-\mathrm{x}} \mathrm{v}_{\mathrm{x}} \mathrm{S}_{4}$ at $4,2 \mathrm{~K}$ [3]

d. Manganite Perovskite $\operatorname{Pr}_{0,7} \mathrm{Ca}_{0,3} \mathrm{MnO}_{3}$ at $100 \mathrm{~K}$ [5]

It can be seen from figure 1 that $\rho_{\mathrm{B}} / \rho_{\mathrm{B}=0}$ values in all observed nonmagnetic and magnetic CMR materials are comparable and demonstrate similar tendency to decrease in a low magnetic field $(\mathrm{B}<1 \mathrm{~T})$.

It is important to note that all observed nonmagnetic semiconductors demonstrate CMR at relatively low temperatures below $4 \mathrm{~K}$ with the exception of $\mathrm{p}-\mathrm{Ge}(\mathrm{Hg})$ demonstrating the same phenomenon at $\mathrm{T}=35 \mathrm{~K}$. It also should be stressed that all CMR nonmagnetic semiconductors are of p-type whereas magnetic semiconductors are both of $\mathrm{p}$ - and n-type.

In high magnetic field $(\mathrm{B}>4 \mathrm{~T}) \mathrm{p}-\mathrm{Hg}_{1-\mathrm{x}} \mathrm{Mn}_{\mathrm{x}} \mathrm{Te}$ and $\mathrm{p}$ $\mathrm{InSb}(\mathrm{Mn})$ demonstrate the increase of resistivity with magnetic field increase, i.e. Positive Magnetoresistance.

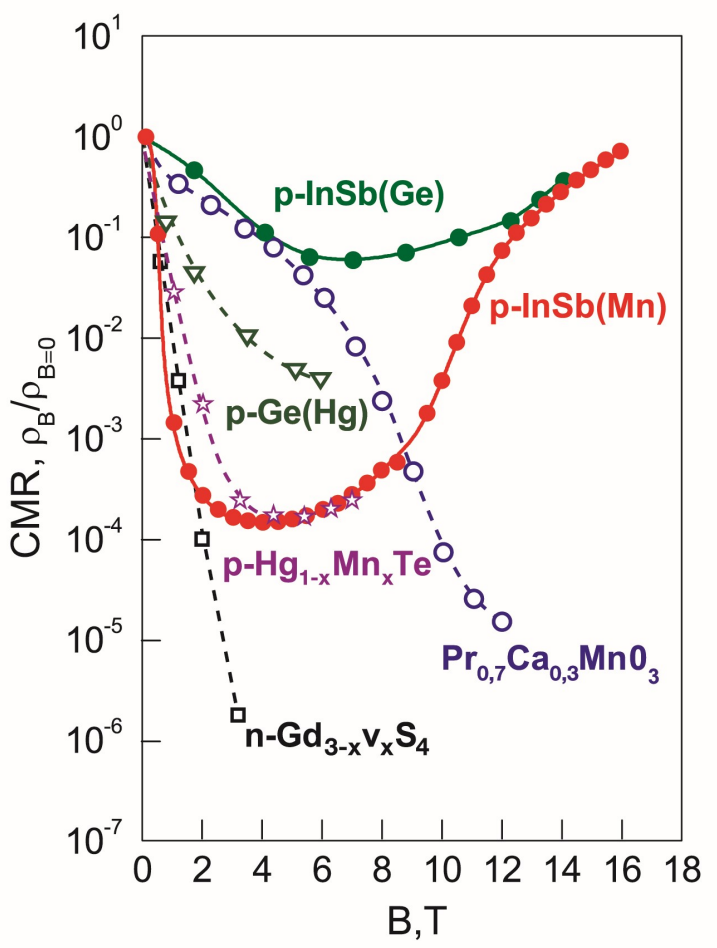

Fig. 1. CMR dependence on magnetic field in nonmagnetic semiconductors and magnetic materials. Dots on solid lines indicate our experimental results in nonmagnetic semiconductors $\mathrm{p}$-InSb(Ge) and $\mathrm{p}$-InSb(Mn). Dashed lines are results for magnetic materials adopted from Ref. [3-5, 9].

\subsection{Charge carrier concentration influence on CMR}

Figure 2 demonstrates the dependence of CMR on charge carrier concentration (n) in the following materials: NMS - $\mathrm{Ge}(\mathrm{Ga}), \mathrm{InSb}(\mathrm{Ge})$ and $\mathrm{InSb}(\mathrm{Mn})$; DMS - p- $\mathrm{Hg}_{1-\mathrm{x}} \mathrm{Mn}_{\mathrm{x}} \mathrm{Te}$ [4]; MS - $\mathrm{n}-\mathrm{Gd}_{3-\mathrm{x}} \mathrm{v}_{\mathrm{x}} \mathrm{S}_{4}$ [3] and EuSe[11]. It can be seen from figure 2 that all CMR materials we study demonstrate similar behavior being the function of charge carrier concentration. At charge carrier concentration below the critical concentration of metal-insulator transition $\left(\mathrm{n}<\mathrm{n}_{\mathrm{cr}}\right)$ the relation $\rho_{\mathrm{B}} / \rho_{\mathrm{B}=0}$ first declines with the decrease of charge carrier concentration, reaches its minimum and finally increases with the decrease of charge carrier concentration.

Figure 2 also shows piezoresistance $\rho_{\chi} / \rho_{\chi=0}$ as a function of charge carrier concentration for the following p-type NMS materials, i.e. p-Ge(Ga), p-InSb(Ge) and p$\mathrm{InSb}(\mathrm{Mn})$. Here the maximal piezoresistance value $\rho_{\chi} / \rho_{\chi=0}$ coincides with the minimal value of $\rho_{\mathrm{B}} / \rho_{\mathrm{B}=0}=\mathrm{f}(\mathrm{n})$ for all observed semiconductors.

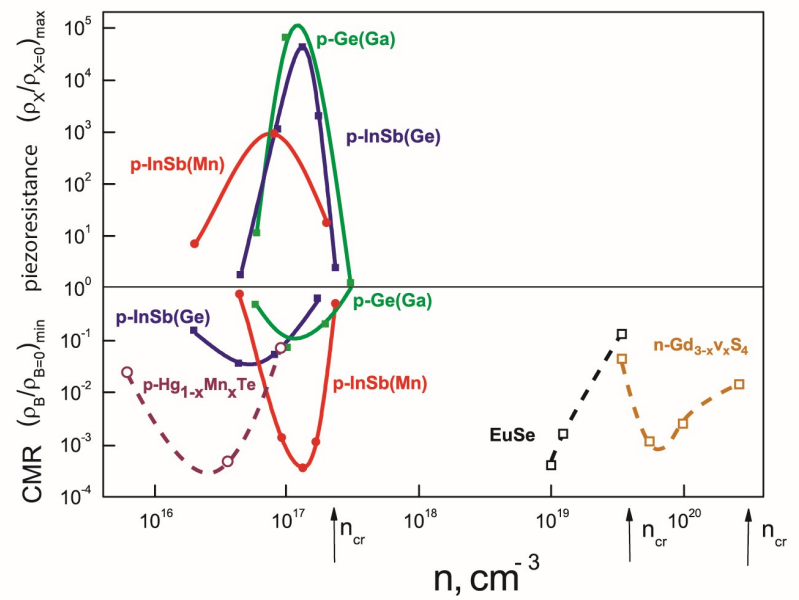

Fig. 2. $\operatorname{CMR}\left(\rho_{\mathrm{B}} / \rho_{\mathrm{B}=0)}\right.$ dependence on charge carrier concentration in magnetic materials, nonmagnetic semiconductors and piezoresistance $\left(\rho_{\chi} / \rho_{\chi=0}\right)$ vs $n$ in $p$-Ge and p-InSb crystals. Both $\rho_{\mathrm{B}} / \rho_{\mathrm{B}=0}$ and $\rho_{\chi} / \rho_{\chi}=0$ dependencies on concentration (n) for $\mathrm{p}-\mathrm{Ge}$ and $\mathrm{p}$-InSb represent our experimental results. Dashed lines are CMR results adopted from Ref. [3,4,11].

\subsection{CMR temperature dependence}

CMR temperature dependence in nonmagnetic and magnetic materials is demonstrated in figure 3 . It can be seen that $\rho_{\mathrm{B}} / \rho_{\mathrm{B}=0}$ exponentially declines with materials cooling in all studied types of semiconductors. The difference is that in nonmagnetic semiconductor $\mathrm{InSb}(\mathrm{Mn})$ at manganese concentration $\mathrm{N}_{\mathrm{Mn}}=1,7 \times 10^{17} \mathrm{~cm}^{-3}$ the minimal value $\rho_{B} / \rho_{B=0}=10^{-4}$ is reached within the millikelvine temperature range, whereas in diluted magnetic semiconductor $\mathrm{p}-\mathrm{Hg}_{1-\mathrm{x}} \mathrm{Mn}_{\mathrm{x}} \mathrm{Te}$ [4] the same CMR effect is observed at higher temperature level which is above $2 \mathrm{~K}$. 


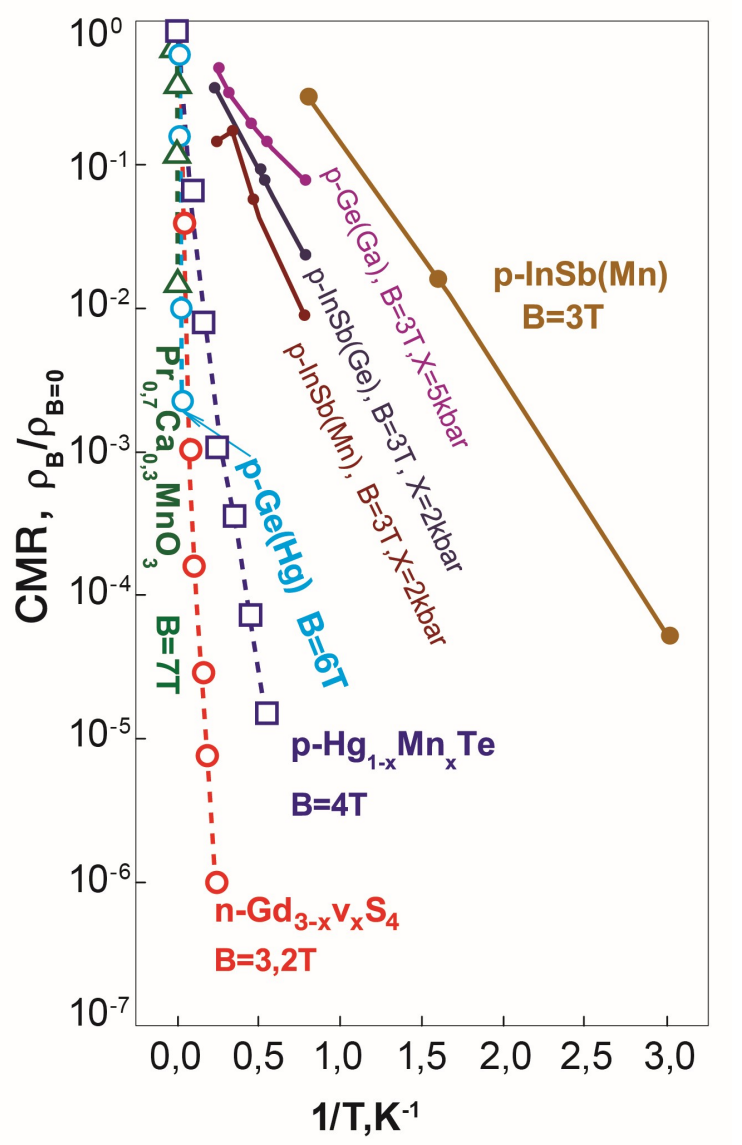

Fig. 3. CMR temperature dependence for nonmagnetic p-InSb and $\mathrm{p}-\mathrm{Ge}$ crystal semiconductors (dots on solid lines -our experimental results) and magnetic materials (dashed linesresults adopted from the Ref. [3-5, 9]).

\subsection{The Hall effect}

To get information concerning the charge carrier contribution into the CMR effect we compare the Hall effect in NMS and DMS. Figure 4 depicts the Hall constant dependence on magnetic field in nonmagnetic InSb crystal doped with $\mathrm{Mn}$ at $\mathrm{N}_{\mathrm{Mn}}=1,5 \times 10^{17} \mathrm{~cm}^{-3}$ and Diluted Magnetic Semiconductor $\mathrm{p}-\mathrm{Hg}_{1-\mathrm{x}} \mathrm{Mn}_{\mathrm{x}} \mathrm{Te}$ [12], where the concentration of $\mathrm{Mn}$ is $10^{4}$ higher. It is clearly seen that both semiconductors demonstrate similar features in magnetic field, i.e. negative Hall constant in low magnetic field and positive Hall constant within the high magnetic field range. The difference between the observed NMS and DMS is that in $\operatorname{InSb}(\mathrm{Mn})$ crystal the Hall constant sign inversion occurs at $\mathrm{B} \sim 1 \mathrm{~T}$ but in $\mathrm{p}$ $\mathrm{Hg}_{1-\mathrm{x}} \mathrm{Mn}_{\mathrm{x}} \mathrm{Te}$ the inversion is revealed at $\mathrm{B} \sim 5 \mathrm{~T}$.

Such Hall constant behavior demonstrates the same tendency of the Hall constant sign inversion and occurs in both types of semiconductors. Here the inversion under different levels of magnetic field could be explained by a large difference in effective masses of electrons and holes which is supported by the fact that $\mathrm{InSb}$ crystals have large hole/electron masses ratio $\left(m_{\mathrm{h}} / m_{\mathrm{e}}=0,5 / 0,01 \sim 50\right)[14]$.

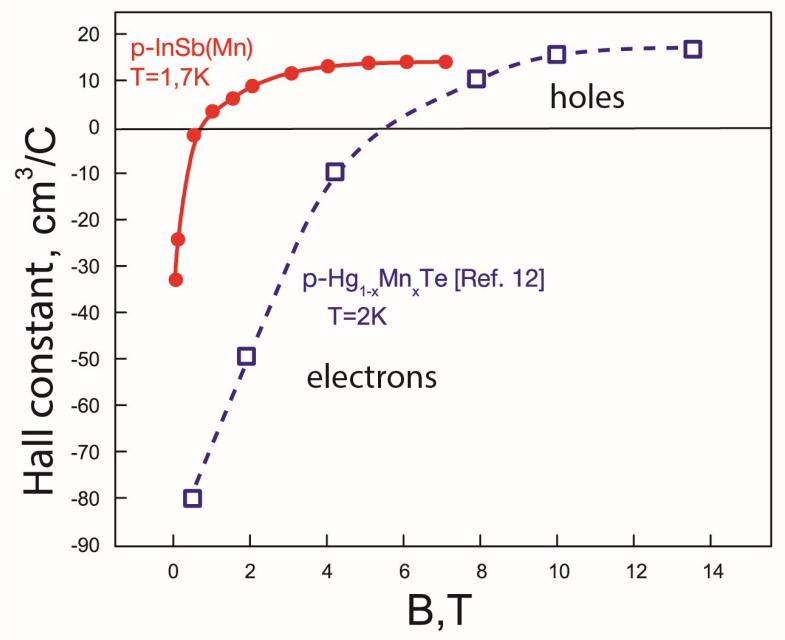

Fig. 4. The Hall constant vs magnetic field in nonmagnetic p$\mathrm{InSb}(\mathrm{Mn})$ crystal (solid line- represents our results) and DMS $\mathrm{p}-\mathrm{Hg}_{1-\mathrm{x}} \mathrm{Mn}_{\mathrm{x}} \mathrm{Te}$ (dashed line-results adopted from [12]).

\section{Discussion}

Having summarized the revealed above qualitative and quantitative CMR and the Hall constant coincidences in nonmagnetic semiconductors and magnetic materials we have come to the following conclusion:

1 It can be suggested that the content of magnetic ions is not the only factor which influences CMR which can be seen from CMR comparison of nonmagnetic InSb doped with manganese at $\mathrm{N}_{\mathrm{Mn}} \sim 10^{17} \mathrm{~cm}^{-3}$ and $\mathrm{p}$ $\mathrm{Hg}_{1-\mathrm{x}} \mathrm{Mn}_{\mathrm{x}} \mathrm{Te}$ or Magnetic Perovskite $\mathrm{Pr}_{0,7} \mathrm{Ca}_{0,3} \mathrm{MnO}_{3}$, where manganese concentration is $10^{5}-10^{6}$ times higher.

2 It is also questionable whether the magnetic type of the CMR material, i.e. ferromagnetic or antiferromagnetic, could be considered the factor which influences NM value.

3 The fact that NM value increases on the insulator side of MIT supports the key role of the charge carrier concentration and the interaction of these charge carriers in producing NM effect.

In this view, the concept of excitonic insulator model (EI model) seems universal for the description of the CMR phenomenon in both magnetic and nonmagnetic materials. It has been suggested [15] that if electron-hole binding energy $E_{B}$ in semiconductors exceeds the gap energy $E_{g}$, a new phase, which is called excitonic insulator, is formed. First the EI model was introduced for the interpretation of resistivity anomalies in $\mathrm{TmSe}_{1}$. ${ }_{x} \mathrm{Te}_{\mathrm{x}}$ [16]. The peak of resistivity with increasing pressure in this material was explained as the sequence of pressure-induced transitions from normal semiconductor to excitonic insulator, and then to normal semimetal. We can suggest that under external magnetic field excitons in CMR materials dissociate and we observe transition from EI to normal semiconductor i.e. the CMR effect.

We propose that in the framework of EI model the EI phase is formed in CMR materials as the result of JahnTeller distortion. In DMS and MS Jahn-Teller (JT) distortion is caused by the host magnetic ions, whereas 
in NMS we see several possibilities to introduce JT distortion. It can be supposed that in $\mathrm{p}-\mathrm{InSb}(\mathrm{Mn})$ crystals JT distortion can be caused by Manganese impurity ions while in $\mathrm{p}-\mathrm{Ge}(\mathrm{Hg})$ the JT distortion could be induced by the deep impurity center of Mercury. As for uniaxially stressed $\mathrm{p}-\mathrm{InSb}(\mathrm{Ge})$ and $\mathrm{p}-\mathrm{Ge}(\mathrm{Ga})$, i.e. semiconductors doped with shallow impurities, JT distortion could appear when we apply uniaxial stress to crystals [17].

In spite of conflicting theories concerning the nature of excitonic insulator its application to the CMR phenomenon description seems to be the important point in the explanation of this phenomenon in both magnetic and nonmagnetic materials.

\section{Conclusion}

Colossal Magnetoresistance in nonmagnetic semiconductors and magnetic materials observed under magnetic field depends on charge carriers concentration and temperature. The revealed qualitative and quantitative coincidence of CMR behaviour in magnetic and nonmagnetic materials highlights the common nature of the CMR phenomenon in these types of materials. The interpretation of insulator-metal transition under magnetic field in the framework of the Excitonic Insulator phase model provides with the possibility to integrate the description of the conductivity transition from EI phase to conducting state for both magnetic and nonmagnetic materials into one model.

The authors acknowledgethe support of National Magnetic Field Laboratory, National Science Foundation Cooperative Agreement No. DMR- 0654118, the State of FL the support by Alexander von Humboldt Foundation, Germany and the support by FAPESP, Brasil. We are grateful to S.W. Tozer and W.A. Coniglio for help with the ${ }^{3} \mathrm{He}-{ }^{4} \mathrm{He}$ dilution refrigerator measurements. We are also grateful A.B. Henriques and N.F. Oliveira for help with ${ }^{3} \mathrm{He}$ cryostat measurements and Prof. W.Gey for help with Bitter magnet measurements.

\section{References}

1. Lord Kelvin, Proc. of the Royal Soc. of London. 8, 546 (1857)

2. D. Chiba, M. Sawicki, Y. Nishitani, Y. Nakatani, F.Matsukura, H. Ohno, Nature. 455, 515 (2008)

3. T. Penney, F. Holtzberg, L.I. Tao, S.von Molnar, AIP Conf. Proc. Magnetizm and Magnetic Materials (ed. C.D. Graham and J.J. Rhyne). №18, 908 (1973)

4. T. Wojtowicz, A. Mycielski, Acta Phys. Polon. A67, 363(1985)

5. Y. Tokura, Physics Today. 56, 50 (2003)

6. E.L. Nagaev, Colossal Magnetoresistance and Phase Separation in Magnetic Semiconductors (Imperial College Press, London, 2002)

7. A. Moreo, E. Dagotto, Science. 283, 2034 (1999)

8. S.A. Obukhov. Phys. Status Solidi. B223, 535 (2001)
9. S.D. Ganichev, H. Ketterl, W. Prettl, I.A. Merkulov, V.I. Perel, I.N. Yassievich, A.V. Malyshev, Phys. Rev. B63, 201204(2001)

10. S.A. Obukhov, S.W. Tozer, W.A. Coniglio, Sci. Rep. 5, 13451(2015)

11. S.A. Obukhov, Phys. Status Solidi. B242, 1298 (2005)

12. A.V. Germanenko, L.P. Zverev, V.V. Kruzhaev, G.M. Min'kov, O.E. Rut', Soviet.Phys.Techn. Semicond. 20, 80 (1984)

13. Y. Shapira, S. Foner, N.F. Oliveira jr, T.B. Reed, Phys. Rev. B10, 4765 (1974)

14. D. Bimberg, Semiconductors (Springer, Berlin 1982)

15. B.I. Halperin and T.M. Rice. Rev. Mod. Phys. 40, 755 (1968)

16. J. Neueschwander, P. Wachter, Phys. Rev. B41, 12693 (1990)

17. G.L. Bir, JETP. 51, 556 (1966) 